\title{
EVALUACIÓN Y DIAGNÓSTICO DE ESTRUCTURAS DE USO PÚBLICO UTILIZANDO TÉCNICAS DINÁMICAS
}

\author{
PERALTA, MARÍA HAYDÉE \\ Ing. Civil \\ Fac. de Ing. UNICEN \\ Bs. As.; Argentina \\ mperalta@ fio.unicen.edu.ar
}

BACCHIARELLO, RAÚL

Ing. Civil

Fac. de Ing. UNICEN

Bs. As.; Argentina

rabac9@hotmail.com

\author{
PICO, LEONEL OSVALDO \\ Ing. Civil \\ Fac. de Ing. UNICEN \\ Bs. As.; Argentina \\ lpico@fio.unicen.edu.ar
}

\author{
ERCOLI, NORMA LUJÁN \\ Ing. Civil \\ Fac. de Ing. UNICEN \\ Bs. As.; Argentina \\ nercoli@fio.unicen.edu.ar
}

\section{RESUMEN}

En el presente trabajo se expone la experiencia realizada por los autores, en el marco de un convenio entre la Facultad de Ingeniería de la Universidad Nacional del Centro de la Provincia de Buenos Aires y el Municipio de Olavarría. Su objetivo fue la evaluación, diagnóstico y propuesta de mantenimiento y/o reparaciones de estructuras de uso público existentes en la ciudad de Olavarría. Para ello, se requirió el análisis de edificios que habían cambiado de destino de utilización. El requerimiento se debió a la percepción de importantes vibraciones durante el uso habitual.

A tal fin, se efectuaron mediciones de vibraciones para determinar las frecuencias naturales y las amplitudes de desplazamientos, velocidades y aceleraciones, lo que posibilitó evaluar la flexibilidad estructural. Los estándares internacionales establecen límites de los parámetros dinámicos para considerar un comportamiento estructural adecuado. Los resultados de las mediciones vibratorias fueron comparados con estos límites, lo cual permitió realizar el diagnóstico del comportamiento, que en algunos casos, motivó una posterior intervención o limitación de uso.

En el trabajo se muestran los análisis realizados sobre un gimnasio, dos salones bailables y el Teatro Municipal de la ciudad. Los resultados obtenidos indican que el análisis dinámico constituye una valiosa técnica de evaluación y diagnóstico de estructuras destinadas a uso público. Esto permite establecer, en servicio, si el comportamiento estructural es adecuado desde el punto de vista de la seguridad requerida para cada caso, o determinar intervenciones en caso contrario.

Palabras-clave: estructuras, evaluación, técnicas dinámicas, intervenciones.

\section{ABSTRACT}

In this work, the experience carried out by the authors is presented, within the framework of an agreement between the Faculty of Engineering of the National University of the Center of the Province of Buenos Aires and the Municipality of Olavarría. Its purpose was the evaluation, diagnosis and proposal of maintenance and / or repairs of public use structures, existing in the city of Olavarría. It required the analysis of buildings that had changed their destination such as gyms, dance halls, Municipal Theater of the city. The requirement was due to the perception of vibrations, in some cases, during habitual use.

For this, vibration measurements were made to determine the natural frequencies and the amplitudes of displacements, velocities and accelerations, which made it possible to evaluate the structural flexibility. International standards establish dynamic parameter limits to consider adequate structural behavior. The results of the vibratory measurements were compared with these limits, which allowed the diagnosis of behavior, which in some cases, led to a subsequent intervention or limitation of use.

This work shows the analysis of a gym, two dance halls and the Municipal Theater of the city. The results obtained indicate that dynamic analysis is a valuable technique for evaluation and diagnosis of structures intended for public use. This allows to establish, in service, if the structural behavior is adequate from the point of view of the security required for each case, or to determine interventions in the opposite case.

Keywords: structures, evaluation, dynamic techniques, interventions. 


\section{INTRODUCCIÓN}

La medición y el análisis de vibraciones son utilizados en conjunto con otras técnicas para la evaluación del comportamiento estructural, constituyendo esta metodología una herramienta útil para el análisis de la integridad estructural (PERALTA, ERCOLI, 2005; PERALTA, ERCOLI, PICO, BACCHIARELLO, LA MALFA, CORREA, 2008, PERALTA, ERCOLI, PICO, LA MALFA, 2009).

Las características dinámicas de las estructuras (CLOUGH, PENZIEN, 1993), pueden usarse como indicadores de su flexibilidad. Esto permite la evaluación del comportamiento y del daño estructural progresivo en el tiempo. Este daño afecta la rigidez inicial y origina una mayor flexibilidad de la estructura y, por consiguiente, causa una mayor vulnerabilidad frente a las acciones dinámicas. De acuerdo con los resultados de las mediciones, la auscultación periódica conduce a adoptar medidas correctivas para que la estructura continúe con las prestaciones establecidas en el proyecto original.

Los códigos de estructuras establecen pautas para efectuar las mencionadas tareas de control con la finalidad de garantizar la vida en servicio de las estructuras, según su destino de uso (RICHART, 1970; MACINANTE, 1984; RAO, 2018). La prevención en la etapa de utilización debe establecer un programa mínimo de conservación, disponer los recursos suficientes, realizar inspecciones y trabajos de mantenimiento periódicos que permitan sostener en el tiempo asignado de vida útil una prestación acorde a la establecida (HUSNI, 2007). El grado de deterioro observado en estructuras que incluyen, en el peor de los casos, los colapsos ocurridos, ha influido en la concientización de la importancia de un monitoreo y mantenimiento de estas estructuras.

En el presente trabajo se expone la experiencia realizada por los autores, en el marco de un convenio entre la Facultad de Ingeniería de la Universidad Nacional del Centro de la Provincia de Buenos Aires y el Municipio de Olavarría. Su objetivo fue la evaluación, diagnóstico y propuesta de mantenimiento y/o reparaciones de estructuras de uso público existentes en la ciudad de Olavarría. Para ello, se requirió el análisis de edificios que, en algunos casos, habían cambiado de destino de utilización y, a la percepción de importantes vibraciones durante el uso habitual.

En el trabajo se muestran los análisis realizados sobre un gimnasio, dos salones bailables y el Teatro Municipal de la ciudad. Los resultados obtenidos indican que el análisis dinámico constituye una valiosa técnica de evaluación y diagnóstico de estructuras destinadas a uso público. Esto permite establecer el grado de seguridad del comportamiento y la toma de decisiones en cuanto a la necesidad de intervenciones si correspondiera.

\section{EVALUACIÓN DEL COMPORTAMIENTO ESTRUCTURAL A TRAVÉS DE VIBRACIONES}

Los niveles aceptables de vibración (RAO, 2018) se especifican en términos de la respuesta de un sistema de un grado de libertad no amortiguado que experimenta vibración armónica. Los límites se muestran en un gráfico, llamado nomograma de vibración, que muestra las variaciones de amplitudes de desplazamiento, de velocidad y de aceleración en función de la frecuencia de vibración. Para el movimiento armónico, el desplazamiento, la velocidad y la aceleración están dadas por las ecuaciones (1), (2) y (3), respectivamente.

$$
\begin{gathered}
x(t)=X \operatorname{sen} \omega t \\
v(t)=\dot{x}(t)=\omega X \cos \omega t=2 \pi f X \cos 2 \pi f t \\
a(t)=\ddot{x}(t)=-\omega^{2} X \operatorname{sen} \omega t=-(2 \pi f)^{2} X \operatorname{sen} 2 \pi f t
\end{gathered}
$$

donde $\omega$ es la frecuencia circular en rad/s, $f$ es la frecuencia lineal en Hz y $X$ es la amplitud del desplazamiento. Las amplitudes del desplazamiento $(X)$, la velocidad $\left(v_{\text {máx }}\right)$ y la aceleración $\left(a_{\text {máx }}\right)$ están relacionadas por las ecuaciones $(4)$ y (5).

$$
\begin{gathered}
v_{\text {máx }}=|2 \pi f X| \\
a_{\text {máx }}=\left|-(2 \pi f)^{2} X\right|=2 \pi f v_{\text {máx }}
\end{gathered}
$$

Para linealizar las ecuaciones (4) y (5) se aplican los logaritmos, resultado las ecuaciones (6) y (7), respectivamente.

$$
\ln v_{\text {máx }}=\ln |2 \pi f X|=\ln 2 \pi f+\ln X
$$




$$
\ln a_{\text {máx }}=\ln \left|-(2 \pi f)^{2} X\right|=\ln \left|2 \pi f v_{\text {máx }}\right|=\ln 2 \pi f+\ln v_{\text {máx }}
$$

Cuando se despeja la amplitud de la velocidad máxima de la ecuación (7) se obtiene la ecuación (8).

$$
\ln v_{\text {máx }}=-\ln 2 \pi f+\ln a_{\text {máx }}
$$

Se puede observar que para un valor constante de desplazamiento (ln $X$, ordenada al origen), la ecuación (6) representa la velocidad máxima en función de la frecuencia con pendiente positiva, +1 . Similarmente, para un valor constante de aceleración (ln $a_{m a ́ x}$, ordenada al origen) la ecuación (8) muestra la velocidad máxima en función de la frecuencia con pendiente negativa, -1 . Estas ecuaciones se pueden graficar en un nomograma de vibraciones tal como el mostrado en la figura 1. Así, con los límites impuestos por los códigos, normativas y bibliografía de referencia (MACINANTE, 1984; RICHART, 1970; ISO DP 4866, 1975; ISO 2631, 1974; ISO 2372, 1974; STEFFENS, 1966) se establecen criterios de diagnóstico del comportamiento estructural y mecánico para diferentes destinos y usos. Por su parte, la figura 2 (RADES, 1994) muestra un criterio adicional de evaluación en función de la amplitud de velocidad vibratoria.

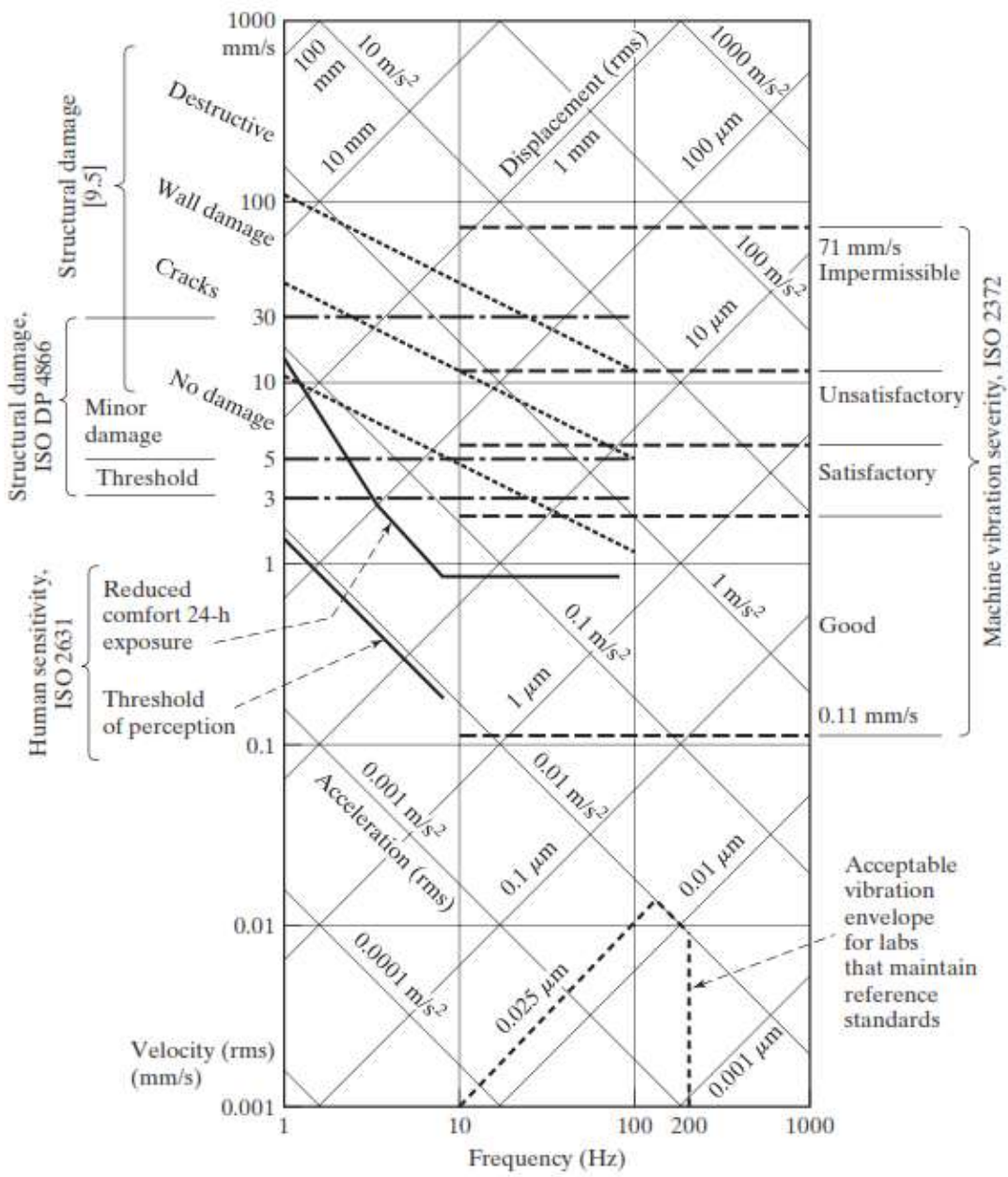

Figura 1. Nomograma de vibraciones.

\begin{tabular}{|c|c|l|}
\hline Range & $\begin{array}{c}\text { rms velocity, } \\
\mathrm{mm} / \mathrm{s}\end{array}$ & \multicolumn{1}{|c|}{ Effect } \\
\hline I & below 2.5 & damages not possible \\
\hline II & $2.5-5.0$ & damages very improbable \\
\hline III & $5.0-10.0$ & damages not probable \\
\hline IV & over 10 & $\begin{array}{l}\text { damages possible } \\
\text { stress check necessary }\end{array}$ \\
\hline
\end{tabular}

Figura 2. Límites vibratorios. 
El criterio de Rades toma como límite de velocidad rms $10 \mathrm{~mm} / \mathrm{s}$, independientemente de la frecuencia, para establecer la necesidad de evaluación estructural por posibles daños.

\section{ESTRUCTURAS EVALUADAS Y METODOLOGÍA DE MEDICIÓN}

Se presentan cuatro de las estructuras de uso público analizadas a partir de lo indicado en el ítem anterior, a saber: un gimnasio, dos salones bailables y un teatro.

En dichas estructuras se determinaron los espectros vibratorios que fueron obtenidos con un analizador espectral (FFT) modelo CSI 1900 que cuenta con un acelerómetro piezoeléctrico modelo CSI 320. Se adoptaron los puntos de medición concordantes con el modo flexional de vibración. Se obtuvieron las amplitudes vibratorias y frecuencias predominantes (FFT) en la dirección flexional vertical. Las mediciones se efectuaron con las estructuras bajo carga estática y bajo la excitación que provocan personas saltando.

\subsection{Gimnasio}

La estructura del gimnasio posee un esquema de losas de hormigón armado apoyadas sobre vigas carteladas y columnas. La figura 3 muestra el entrepiso del gimnasio y la disposición de los sensores vibratorios. De acuerdo con las observaciones visuales realizadas, la estructura no presenta discontinuidades a la vista que justifiquen especial atención en determinado sector al efectuar las mediciones.


Figura 3: Entrepiso del gimnasio y mediciones efectuadas.

\subsection{Salón bailable 1}

La estructura del entrepiso de este salón bailable, que se observa en la figura 4, presenta un esquema diferenciado en dos sectores: una zona recientemente incorporada al edificio, metálica con entablonado de madera, y otra de hormigón armado, correspondiente al edificio original, revestida con cielorraso y piso.
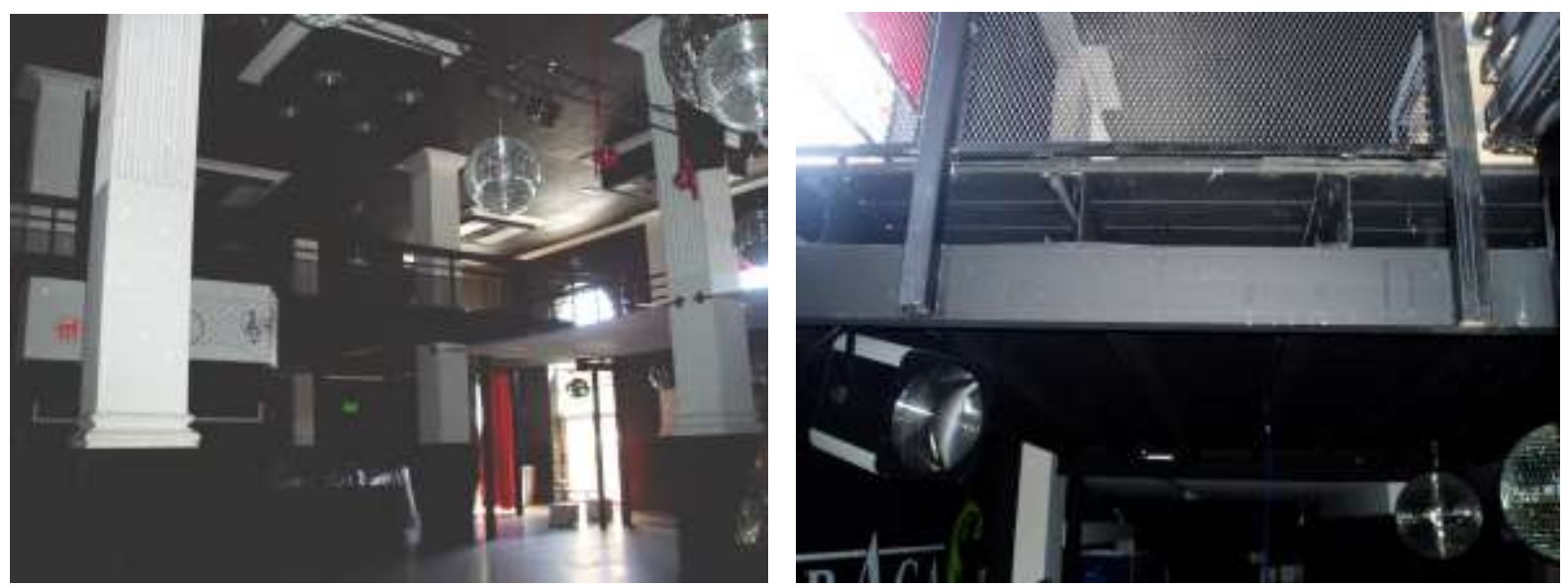

Figura 4: Entrepiso del local bailable 1 y detalle de zona metálica. 


\subsection{Salón bailable 2}

En la figura 5 se muestra la estructura del entrepiso del local bailable formado por vigas de madera con diferentes rigideces por tramos apoyadas sobre paredes de mampostería de ladrillos de antigua data. Se observa que el entrepiso posee un piso tipo flotante. Los tramos extremos poseen refuerzos de perfiles metálicos y el tramo central dispone de chapas que forran la parte inferior de las vigas de madera.
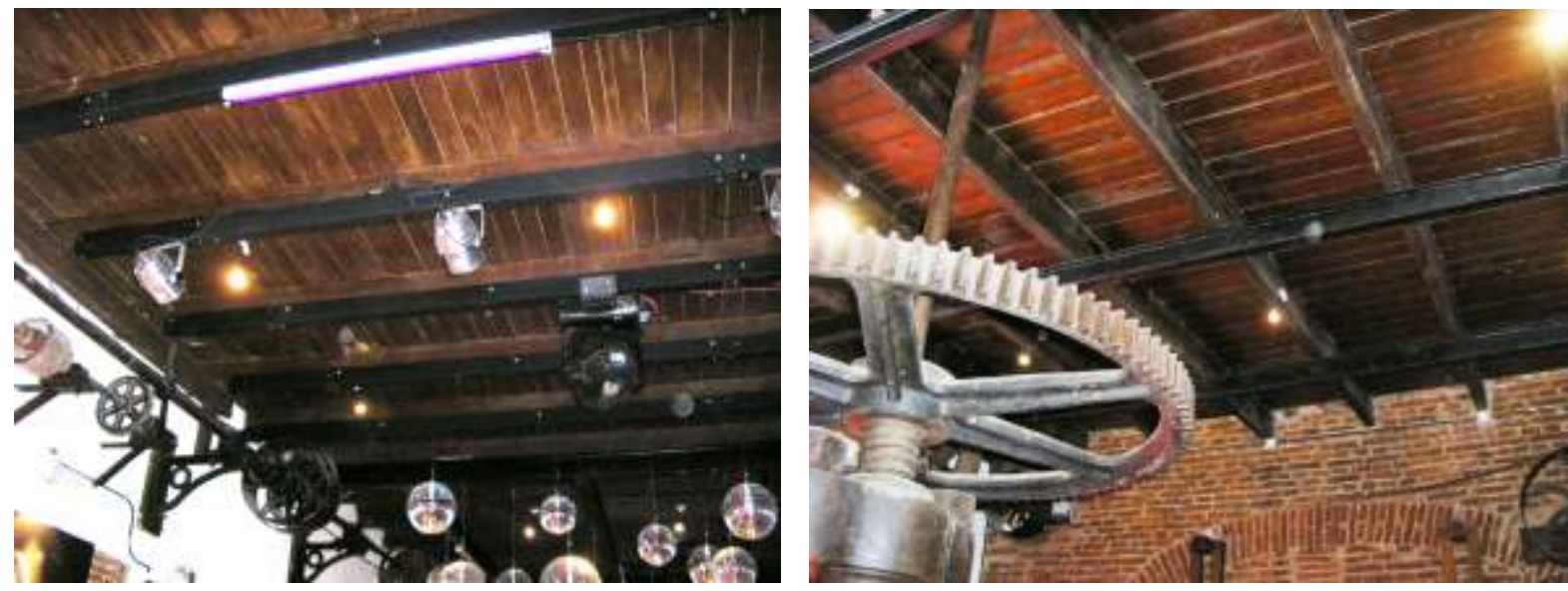

Figura 5: Entrepiso del local bailable 2 y vista de los refuerzos metálicos.

\subsection{Teatro Municipal}

La estructura del teatro, analizada, está formada por 19 reticulados apoyados sobre un muro trasero y por una viga transversal reticulada de $23 \mathrm{~m}$ de luz con un voladizo que sirve de soporte de los palcos de $5 \mathrm{~m}$ de luz. La figura 6 muestra el esquema estructural y una vista de la bandeja del teatro.

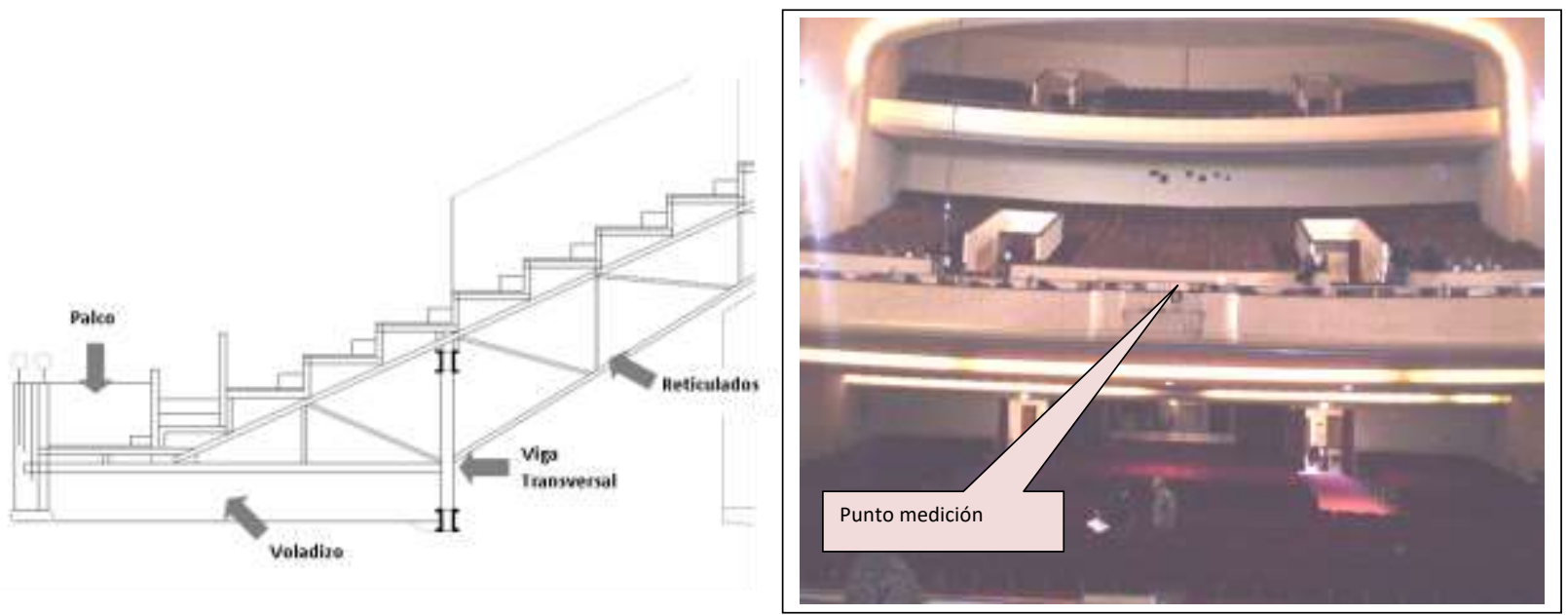

Figura 6: Entrepiso del Teatro Municipal y punto de medición.

\section{RESULTADOS DE LAS MEDICIONES Y DISCUSIÓN}

\subsection{Gimnasio}

La figura 7 muestra los resultados de velocidad $\mathrm{rms}$ en $\mathrm{mm} / \mathrm{s}$ en función de la frecuencia natural en $\mathrm{Hz}$. De esta figura se puede inferir que la frecuencia natural predominante es $12,61 \mathrm{~Hz}$ y que la amplitud de velocidad es $0,576 \mathrm{~mm} / \mathrm{s}$. 


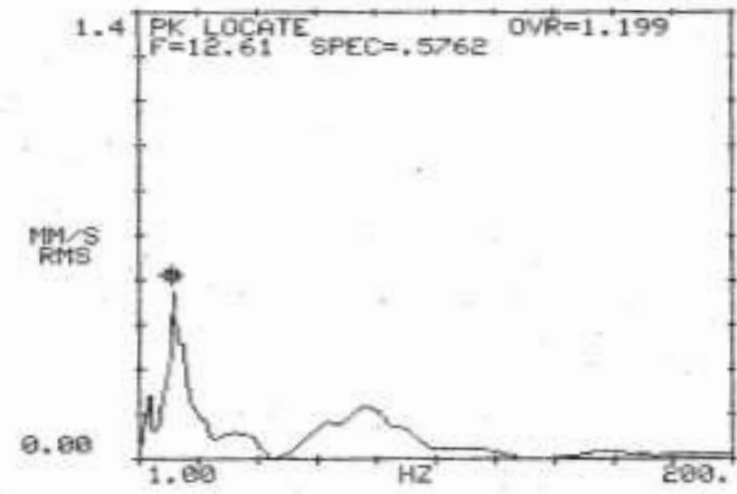

Figura 7: Velocidad en función de la frecuencia natural del entrepiso del gimnasio.

\subsection{Salón bailable 1}

En la figura 8 se muestra el espectro de vibraciones medidas. Los resultados indican que, en la zona metálica, la frecuencia predominante es $3,615 \mathrm{~Hz}$ y que la amplitud de velocidad máxima es $22,60 \mathrm{~mm} / \mathrm{s}$. Por su parte, en las zonas de hormigón armado, la velocidad máxima es menor que el límite de $10 \mathrm{~mm} / \mathrm{s}$ establecido por Rades (figura 2).

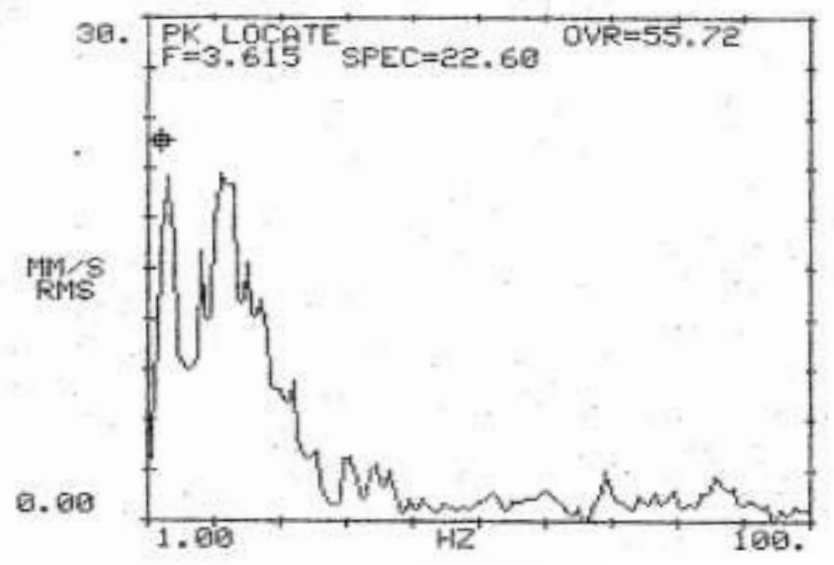

Figura 8: Entrepiso del local bailable 1 y zona analizada.

\subsection{Salón bailable 2}

En la figura 9 se muestra el espectro de vibraciones medidas. Los resultados medidos indican que la frecuencia predominante es $4,25 \mathrm{~Hz}$ y que la amplitud de velocidad máxima es $24,44 \mathrm{~mm} / \mathrm{s}$.

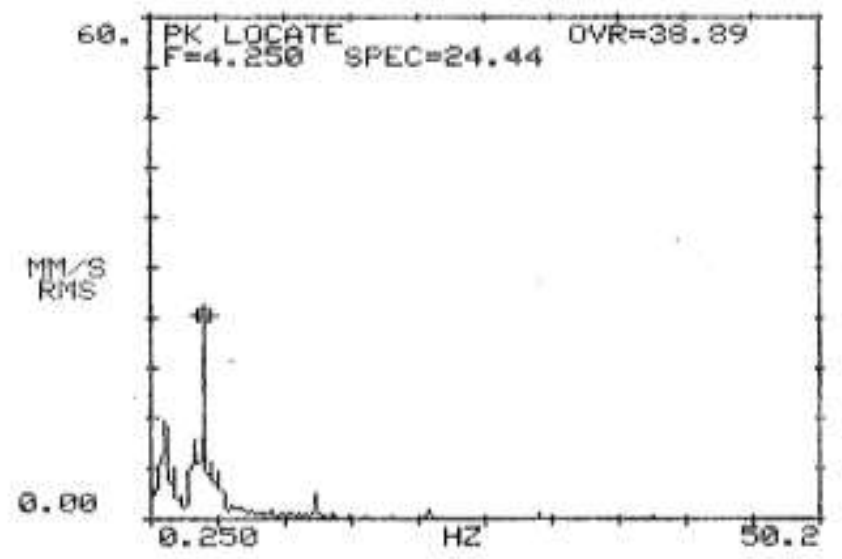

Figura 9: Velocidad en función de la frecuencia natural del entrepiso del salón bailable 2. 


\subsection{Teatro Municipal}

La figura 10 muestra el espectro vibratorio medido. Los resultados indican que la frecuencia natural es $4 \mathrm{~Hz}$ y que la máxima amplitud de velocidad es $19,95 \mathrm{~mm} / \mathrm{s}$.

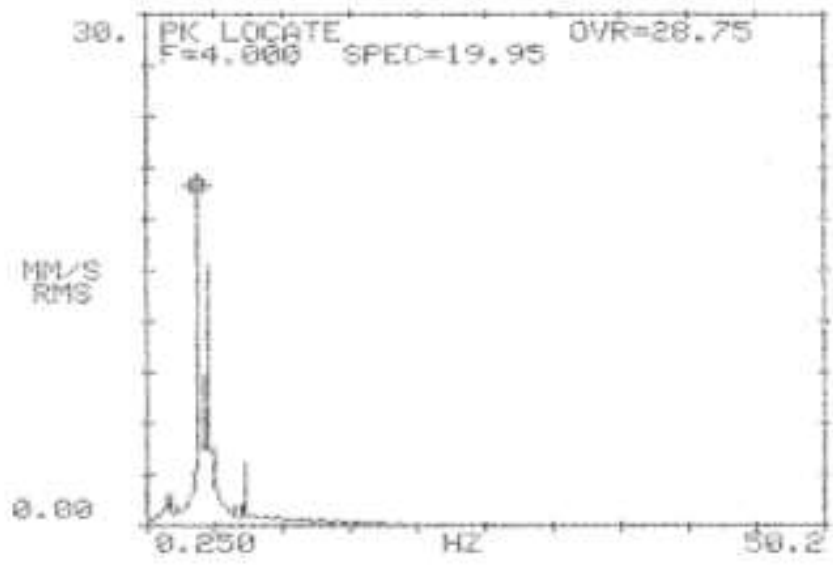

Figura 10: Velocidad en función de la frecuencia natural del entrepiso del Teatro Municipal.

La tabla 1 muestra los resultados dinámicos obtenidos para las cuatro estructuras analizadas. Se muestran los resultados de frecuencia natural y de amplitudes vibratorias máximas de velocidad y el diagnóstico realizados en función del análisis proporcionado por el nomograma de figura 1 y los límites vibratorios de la figura 2 . En el caso del gimnasio se puede afirmar que no existe deterioro estructural, dado que la frecuencia obtenida es menor que $6 \mathrm{~Hz}$ y que la velocidad máxima es mucho menor que $10 \mathrm{~mm} / \mathrm{s}$. En cuanto a los salones bailables 1 y 2 y el Teatro Municipal se puede inferir que las mediciones efectuadas exceden los límites admisibles recomendados.

Tabla 1 - Resultados dinámicos y diagnóstico de las estructuras analizadas.

\begin{tabular}{|c|c|c|c|}
\hline \multirow[b]{2}{*}{ Estructura } & \multicolumn{2}{|c|}{ Resultados dinámicos } & \multirow[b]{2}{*}{$\begin{array}{c}\text { Diagnóstico del comportamiento y patologías } \\
\text { detectadas }\end{array}$} \\
\hline & $\begin{array}{c}\text { Frecuencia } \\
\text { natural }(\mathrm{Hz})\end{array}$ & $\begin{array}{c}\text { Velocidad } \\
(\mathrm{mm} / \mathrm{s})\end{array}$ & \\
\hline Gimnasio & 12,610 & 0,576 & $\begin{array}{l}\text { Comportamiento adecuado. No hay daño } \\
\text { estructural. No es necesaria una intervención. }\end{array}$ \\
\hline Salón bailable 1 & 3,615 & 22,60 & \multirow{3}{*}{$\begin{array}{l}\text { Comportamiento inadecuado. Alta flexibilidad de } \\
\text { la estructura. Existe posibilidad de daño estructural. }\end{array}$} \\
\hline Salón bailable 2 & 4,250 & 24,44 & \\
\hline Teatro Municipal & 4,000 & 19,95 & \\
\hline
\end{tabular}

De acuerdo con estos resultados, se realizaron las siguientes recomendaciones para las estructuras con comportamientos inadecuados:

- Efectuar relevamientos de la estructuras existentes que permitan identificar las secciones y materiales de los elementos que las constituyen.

- Proyectar y ejecutar refuerzos estructurales que permitan rigidizar convenientemente los entrepisos para habilitar su uso para destinos que involucren las acciones de características dinámicas como las que corresponden a cada caso.

En base a las recomendaciones indicadas se tomaron las siguientes medidas:

- Salón bailable 1: Se inhabilitó el local para ese uso.

- Salón bailable 2: La zona metálica observada como fuera de los límites permitidos para la seguridad estructural fue restringida en su uso solo como lugar de estar.

- Teatro Municipal: Se realizó el relevamiento y posterior cálculo de refuerzos estructurales que permitieron arribar a un comportamiento dentro de los límites de uso seguro para la estructura. 


\section{CONCLUSIONES}

Los resultados obtenidos para los casos estudiados permiten destacar la validez de la evaluación del comportamiento estructural a partir de la determinación de las características y sus respuestas dinámicas.

Asimismo, queda de manifiesto la importancia y necesidad de la evaluación dinámica de las estructuras en la etapa de diseño para evitar comportamientos inadecuados durante su vida en servicio.

Por lo expuesto el análisis dinámico de las estructuras constituye una técnica apropiada para evaluar daño estructural y/o afectación de la capacidad portante. Esta metodología de análisis dinámico constituye una técnica no destructiva muy práctica en su aplicación, lo cual la hace recomendable fundamentalmente antes y después de una intervención.

\section{REFERENCIAS}

CLOUGH, R. W.; PENZIEN, J. Dynamics of Structures. Second Edition, McGraw-Hill, Inc. 1993.

HUSNI, R. Fallas y prevención en las estructuras de hormigón. Revista Ingeniería Estructural. Año 15, n. 39. 2007.

ISO 2372. INTERNATIONAL ORGANIZATION FOR STANDARDIZATION. Mechanical Vibration of Machines with Operating Speeds from 10 to 200 rev/s. Basis for Specifying Evaluation Standards. 1974.

ISO 2631. INTERNATIONAL ORGANIZATION FOR STANDARDIZATION. Guide for the Evaluation of Human Exposure to Whole-Body Vibration. 1974.

ISO DP 4866. INTERNATIONAL ORGANIZATION FOR STANDARDIZATION. Evaluation and Measurement of Vibration in Buildings. Draft proposal. 1975.

MACINANTE, J. A. Seismic Mountings for Vibration Isolation. Wiley, New York, 1984.

PERALTA, M.; ERCOLI, N. Measuring the Dynamic Properties to Detect Structural Damage. IV International ACI/CANMET. Conference of Quality of Concrete Structures and Recent Advances in Concrete Materials and Testing. Olinda, Pernambuco State. Brazil. Sesion VII Non Destructive Test. pp. 463-475. 2005.

PERALTA, M.; ERCOLI, N.; PICO, L.; BACCHIARELLO, R.; LA MALFA, S.; CORREA, L. Análisis Numérico y Experimental de Vibraciones para la Evaluación del Comportamiento de un Puente. Mecánica Computacional, Vol. XXVII, pp. 2033-2052. 2008.

PERALTA, M.; ERCOLI, N.; PICO, L.; LA MALFA, S. Utilización de Técnicas Experimentales Dinámicas en la Evaluación de Estructuras. $8^{\circ}$ EIPAC, Encuentro de Investigadores y Profesionales Argentinos de la Construcción, Mendoza, Argentina. 2009.

RADES, M. Vibration Limits for Industrial Buildings. The Shock and Vibration Digest, v. 26, n. 3. 1994.

RAO, S. Mechanical Vibrations. Sixth Edition, Pearson Education, Prentice Hall, 2018.

RICHART F. E. JR.; HALL, J. R.; WOODS, R.D. Vibrations of soils and foundations. Prentice Hall. 1970.

STEFFENS, R. J. Some aspects of structural vibration. Proceedings of the Syposium on Vibrations in Civil Engineering. B. O. Skipp (ed.). Butterworths. London, 1966. 